\title{
Majorana fermion states and fractional flux periodicity in mesoscopic $d$-wave superconducting loops with spin-orbit interaction
}

\author{
Guo-Qiao Zha, ${ }^{1,2,{ }^{*}}$ Lucian Covaci, ${ }^{1}$ F. M. Peeters, ${ }^{1}$ and Shi-Ping Zhou ${ }^{2}$ \\ ${ }^{1}$ Departement Fysica, Universiteit Antwerpen, Groenenborgerlaan 171, B-2020 Antwerpen, Belgium \\ ${ }^{2}$ Department of Physics, Shanghai University, Shanghai 200444, China \\ (Received 27 May 2014; revised manuscript received 8 July 2014; published 31 July 2014)
}

\begin{abstract}
We numerically investigate the spin-orbit (SO) coupling effect on the magnetic flux evolution of energy and supercurrent in mesoscopic $d$-wave superconducting loops by solving the spin-generalized Bogoliubov-de Gennes equations self-consistently. It is found that the energy spectrum splits when the SO interaction is involved and the Majorana zero mode can be realized in the [100] edges of square systems for an appropriate SO coupling strength. Superconducting phase transitions appear when the energy gap closes, accompanied by energy jumps between different energy parabolas in the ground state, which provides a possible mechanism to support fractional flux periodicity of supercurrent. Moreover, in the case of rectangular loops with SO coupling, the jumps of the ground-state energy gradually disappear by increasing the ratio of length to height of the sample, and a paramagnetic response with opposite direction of the screening current around zero flux value can occur in such systems.
\end{abstract}

DOI: 10.1103/PhysRevB.90.014522

PACS number(s): 74.20.Rp, 74.25.Ha, 74.78.Na

\section{INTRODUCTION}

Recently, the periodic evolution of the flux-induced current in multiply connected superconducting systems has drawn a lot of attention [1-9], which is a valuable probe into the mechanism of superconductivity and which is the basis for many applications. For unconventional mesoscopic superconductors with gap nodes, such as $d$-wave loops, the doubling of well-known $h c / 2 e$-flux periodicity has been predicted due to the lifted degeneracy in energy and the offset of the transition between different current-carrying states [1,8]. Moreover, unusual breaking of the $h c / 2 e$ periodicity, such as $h c / 4 e$ oscillations and other fractional periodicities (like $h c / 6 e$ and $h c / 8 e$ ) of the critical current, has been reported in a superconducting quantum interference device geometry with high- $T_{c}$ superconductors (HTS) $[10,11]$. The multiple Andreev scattering in grain-boundary Josephson junctions $[10,11]$ and interactions between Cooper pairs and quartet formation [12-14] have been considered theoretically to be the likely origin of the fractional periodicity. More recently, the pair-density wave state was proposed as a candidate ground state of striped cuprate superconductors $[15,16]$ and can sustain a $h c / 4 e$ vortex phase [17-20].

Another system which may support fractional flux quanta is the spin-triplet $p$-wave superconductor [21,22]. In the equal spin pairing $p$-wave state, there are two weakly interacting condensates with Cooper-pair spin configurations. Electrons with spin-up form a single vortex state, while those with spin-down may form a vortex-free state in a mesoscopic situation [22]. Recent cantilever magnetometry measurements of annular mesoscopic samples of $\mathrm{Sr}_{2} \mathrm{RuO}_{4}$ have revealed evidence for the existence of half-quantum vortices in this material [23]. Furthermore, the coexistence of different winding numbers in two superconducting condensates have also been discussed in the context of fractional vortices for simply and

*zgq_1981@shu.edu.cn multiply connected samples [24-28], and similar phenomena can be expected for many other systems [29,30].

In the present work we provide a careful insight into the spin-orbit (SO) coupling effect on flux-dependent ground-state evolution in mesoscopic $d$-wave superconducting noncircular systems. Superconducting systems with strong SO coupling have attracted a great deal of attention recently [31-47]. It is well known that structural inversion asymmetry can be created at surfaces, an interface, or some junction structures. In materials without inversion symmetry, Rashba-type SO interactions lift the spin degeneracy of the electronic bands and generate complex spin textures in the electron Bloch functions. It has been predicted that an additional $p$-wave component can be generated by the surface induced SO coupling in the case of $d$-wave superconductors [31-34]. Furthermore, strong $\mathrm{SO}$ interactions in nodal superconductors can give rise to a nontrivial band topology, leading to topologically protected zero-energy edge states [38-44]. In particular, it has been shown that Rashba SO couping is essential for the appearance of Majorana fermions, which can be realized even if the symmetry of the gap function is spin-singlet dominant [45-47]. These all imply that the SO coupling effect in mesoscopic $d$-wave superconductors may produce very rich phenomena, such as a variety of possible evolutions of flux periodicity in superconducting loops.

In view of the above, we introduce $\mathrm{SO}$ interactions into the effective model Hamiltonian with $d$-wave superconducting order and systematically investigate the SO coupling effect on the flux periodicity evolution in mesoscopic superconducting loops by solving the extended Bogoliubov-de Gennes (BdG) equations $[34,37,45,48]$ in a self-consistent manner. Our numerical analysis concerns the flux-dependent evolution of ground-state energy and circulating current in square and rectangular systems as a function of the SO interaction strength. The energy spectrums with distinct spin split phenomenon are demonstrated correspondingly. For an appropriate strength of SO coupling, the highest occupied current-carrying state can cross the Fermi level at some flux values, and zero-energy 
Majorana edge modes are realized in such systems. As a consequence, the phase transitions between different energy parabolas in the ground-state energy may lead to fractional flux periodic evolution of current in unconventional superconducting loops.

The paper is organized as follows. In Sec. II we present our theoretical formalism. The Majorana fermion states and fractional flux periodicity in mesoscopic loop systems with spin-orbit interactions are discussed in Sec. III. Our results are summarized in Sec. IV.

\section{THEORETICAL APPROACH}

To investigate the flux periodicity in mesoscopic loops of $d$-wave superconductors whose edges are oriented parallel to the [100] direction, we start with the pairing Hamiltonian by assuming nearest-neighbor attraction $V$ for $d$-wave superconducting (DSC) pairing:

$$
\begin{gathered}
\hat{H}=\hat{H}_{0}+\hat{H}_{\mathrm{so}}, \\
\hat{H}_{0}=-\sum_{\langle\mathbf{i}\rangle, \sigma} t_{\mathbf{i j}} \exp \left(i \varphi_{\mathbf{i j}}\right) c_{\mathbf{i} \sigma}^{\dagger} c_{\mathbf{j} \sigma}+\sum_{\mathbf{i}, \sigma}(-\mu) c_{\mathbf{i} \sigma}^{\dagger} c_{\mathbf{i} \sigma} \\
+\sum_{\langle\mathbf{i} \mathbf{j}\rangle}\left(\Delta_{\mathbf{i j}} c_{\mathbf{i} \uparrow}^{\dagger} c_{\mathbf{j} \downarrow}^{\dagger}+\Delta_{\mathbf{i j}}^{*} c_{\mathbf{j} \downarrow} c_{\mathbf{i} \uparrow}\right), \\
\hat{H}_{\mathrm{so}}=V_{\text {so }} \sum_{\mathbf{i}}\left[\left(c_{\mathbf{i} \uparrow}^{\dagger} c_{\mathbf{i}+\vec{e}_{x} \downarrow}-c_{\mathbf{i} \downarrow}^{\dagger} c_{\mathbf{i}+\vec{e}_{x} \uparrow}\right)\right. \\
\left.-i\left(c_{\mathbf{i} \uparrow}^{\dagger} c_{\mathbf{i}+\vec{e}_{y} \downarrow}+c_{\mathbf{i} \downarrow}^{\dagger} c_{\mathbf{i}+\vec{e}_{y} \uparrow}\right)+\text { H.c. }\right],
\end{gathered}
$$

where $t_{\mathbf{i j}}=t$ are the nearest-neighbor hopping integral and $V_{\text {so }}$ is the SO coupling strength. $c_{\mathbf{i} \sigma}\left(c_{\mathbf{i} \sigma}^{\dagger}\right)$ are destruction (creation) operators for electron of $\operatorname{spin} \sigma(\sigma=\uparrow$ or $\downarrow)$, and $\vec{e}_{x, y}$ is the unit vector along the $x$ and $y$ directions. $n_{\mathbf{i} \sigma}=c_{\mathbf{i} \sigma}^{\dagger} c_{\mathbf{i} \sigma}$ is the number operator, and $\mu$ is the chemical potential determining the averaged electron density. The Peierl's phase factor is given by $\varphi_{\mathbf{i j}}=2 \pi / \Phi_{0} \int_{r_{\mathrm{i}}}^{r_{\mathrm{j}}} \mathbf{A}(\mathbf{r}) d \mathbf{r}$ with the flux quantum $\Phi_{0}=h c / e$. We choose a vector potential of the form $\mathbf{A}(\mathbf{r})=(y,-x, 0) \Phi /\left[2 \pi\left(x^{2}+y^{2}\right)\right]$, yielding a flux threading the hole with no magnetic field penetrating the superconductor, where $\phi=(e / h c) \Phi$ measures the flux in units of $h c / e$. The DSC order has the following definition: $\Delta_{\mathrm{ij}}=$ $V\left\langle c_{\mathbf{i} \uparrow} c_{\mathbf{j} \downarrow}-c_{\mathbf{i} \downarrow} c_{\mathbf{j} \uparrow}\right\rangle / 2$. Using the Bogoliubov transformation $c_{\mathbf{i} \sigma}=\sum_{n}\left[u_{\mathbf{i} \sigma}^{n} \gamma_{n \sigma}-\sigma v_{\mathbf{i} \sigma}^{n *} \gamma_{n \bar{\sigma}}^{\dagger}\right]$, the Hamiltonian in Eq. (1) can be diagonalized by solving the resulting $\mathrm{BdG}$ equations self-consistently,

$$
\sum_{\mathbf{j}}^{N}\left(\begin{array}{cccc}
\mathcal{H}_{\mathbf{i j} \uparrow} & V_{1}^{\text {so }} & 0 & \Delta_{\mathbf{i j}} \\
V_{2}^{\text {so }} & \mathcal{H}_{\mathbf{i j} \downarrow} & \Delta_{\mathbf{i j}} & 0 \\
0 & \Delta_{\mathbf{i} \mathbf{j}}^{*} & -\mathcal{H}_{\mathbf{i j} \uparrow}^{*} & V_{3}^{\text {so }} \\
\Delta_{\mathbf{i j}}^{*} & 0 & V_{4}^{\text {so }} & -\mathcal{H}_{\mathbf{i j} \downarrow}^{*}
\end{array}\right)\left(\begin{array}{c}
u_{\mathbf{j} \uparrow}^{n} \\
u_{\mathbf{j} \downarrow}^{n} \\
v_{\mathbf{j} \uparrow}^{n} \\
v_{\mathbf{j} \downarrow}^{n}
\end{array}\right)=E_{n}\left(\begin{array}{c}
u_{\mathbf{i} \uparrow}^{n} \\
u_{\mathbf{i} \downarrow}^{n} \\
v_{\mathbf{i} \uparrow}^{n} \\
v_{\mathbf{i} \downarrow}^{n}
\end{array}\right),
$$

where $\quad \mathcal{H}_{\mathbf{i j} \sigma}=-t_{\mathbf{i j}}-\mu \delta_{\mathbf{i j}}, \quad V_{m}^{\text {so }}=V_{\text {so }}\left\{\left[(-1)^{m+1} \delta_{\mathbf{i}+\vec{e}_{x}, \mathbf{j}}+\right.\right.$ $\left.\left.(-1)^{m} \delta_{\mathbf{i}-\vec{e}_{x}, \mathbf{j}}\right]+\lambda_{m}\left[i\left(\delta_{\mathbf{i}+\vec{e}_{y}, \mathbf{j}}-\delta_{\mathbf{i}-\vec{e}_{y}, \mathbf{j}}\right)\right]\right\}\left(\lambda_{m}=-1\right.$ for $m=1$, 2 and $\lambda_{m}=1$ for $\left.m=3,4\right)$, and $\delta_{\mathbf{i}, \mathbf{j}}$ represents a $\delta$ function. With the open boundary conditions (for which the wave function vanishes on the inner and outer boundaries of the

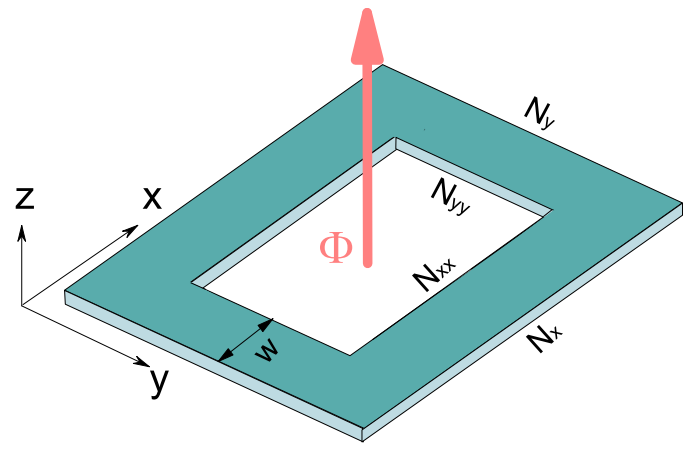

FIG. 1. (Color online) Schematic view of the two-dimensional loop with size $N_{x} \times N_{y}$ and a centered hole of size $N_{x x} \times N_{y y}$, which is threaded by a magnetic field $\Phi$ in the hole. $w$ is the arm width of the sample.

loop) we can get the eigenvalues $\left\{E_{n}\right\}$ with eigenvectors $\left\{u_{\uparrow}^{n}, u_{\downarrow}^{n}, v_{\uparrow}^{n}, v_{\downarrow}^{n}\right\}$. The order parameter $\Delta_{\mathbf{i j}}$ is calculated self-consistently from

$$
\begin{aligned}
\Delta_{\mathbf{i j}}= & \sum_{n} \frac{V}{4}\left(u_{\mathbf{i} \uparrow}^{n} v_{\mathbf{j} \downarrow}^{n *}+u_{\mathbf{j} \downarrow}^{n} v_{\mathbf{i} \uparrow}^{n *}\right. \\
& \left.+u_{\mathbf{i} \downarrow}^{n} v_{\mathbf{j} \uparrow}^{n *}+u_{\mathbf{j} \uparrow}^{n} v_{\mathbf{i} \downarrow}^{n *}\right) \tanh \left(\frac{E_{n}}{2 k_{B} T}\right) .
\end{aligned}
$$

Since the order parameter has to be single valued, the phase difference when circulating the hole once must be $2 \pi q$, where $q$ is the winding number. The current density $J_{\mathbf{i j} \sigma}$ from lattice site $\mathbf{i}$ to $\mathbf{j}$ is

$$
\begin{aligned}
J_{\mathbf{i j} \sigma}= & -4 \frac{e t}{\hbar c} \sum_{n, \sigma} \operatorname{Im}\left(\left\{u_{\mathbf{j} \sigma}^{n} u_{\mathbf{i} \sigma}^{n *} f\left(E_{n}\right)\right.\right. \\
& \left.\left.+v_{\mathbf{j} \sigma}^{n *} v_{\mathbf{i} \sigma}^{n}\left[1-f\left(E_{n}\right)\right]\right\} \exp \left(i \varphi_{\mathbf{i j}}\right)\right),
\end{aligned}
$$

where $f(E)=\left(e^{E / k_{B} T}+1\right)^{-1}$ is the Fermi-Dirac distribution function.

Throughout this work, the distance is measured in units of the lattice constant $a$, the energy in units of $t$, the magnetic flux in units of $\Phi_{0}=h c / e$, and the current density in units of $J_{0}=e t / \hbar c$. In the numerical calculations we take $k_{B}=$ $a=t=1$ for simplicity and only consider the half-filled case, i.e., $\mu=0$. In what follows, we focus on the clean sample as schematically shown in Fig. 1 with a size of $N_{x} \times N_{y}$ and a centered hole of size $N_{x x} \times N_{y y}$, which is threaded by a magnetic field $\Phi$ in the hole. $w=\left(N_{x(y)}-N_{x x(y y)}\right) / 2$ is the arm width of the sample.

\section{FRACTIONAL FLUX PERIODICITY AND MAJORANA FERMION STATES IN EDGES OF THE SAMPLE}

First, we consider a square $d$-wave loop with size $N_{x} \times$ $N_{y}=40 \times 40$ and a centered hole of size $N_{x x} \times N_{y y}=$ $28 \times 28$. In this case, the arm width is $w=6$. In order to determine how the properties of the loop depend on the SO coupling strength $V_{\text {so }}$, we start our investigations with a system with small $V_{\text {so }}$ at zero temperature. The black and red curves in Fig. 2 gives the total energy (a) and the total supercurrent (b) as a function of magnetic flux $\Phi$ for $V_{\text {so }}=0$ (black solid curves) and $V_{\text {so }}=0.2$ (red dashed curves), respectively. The 


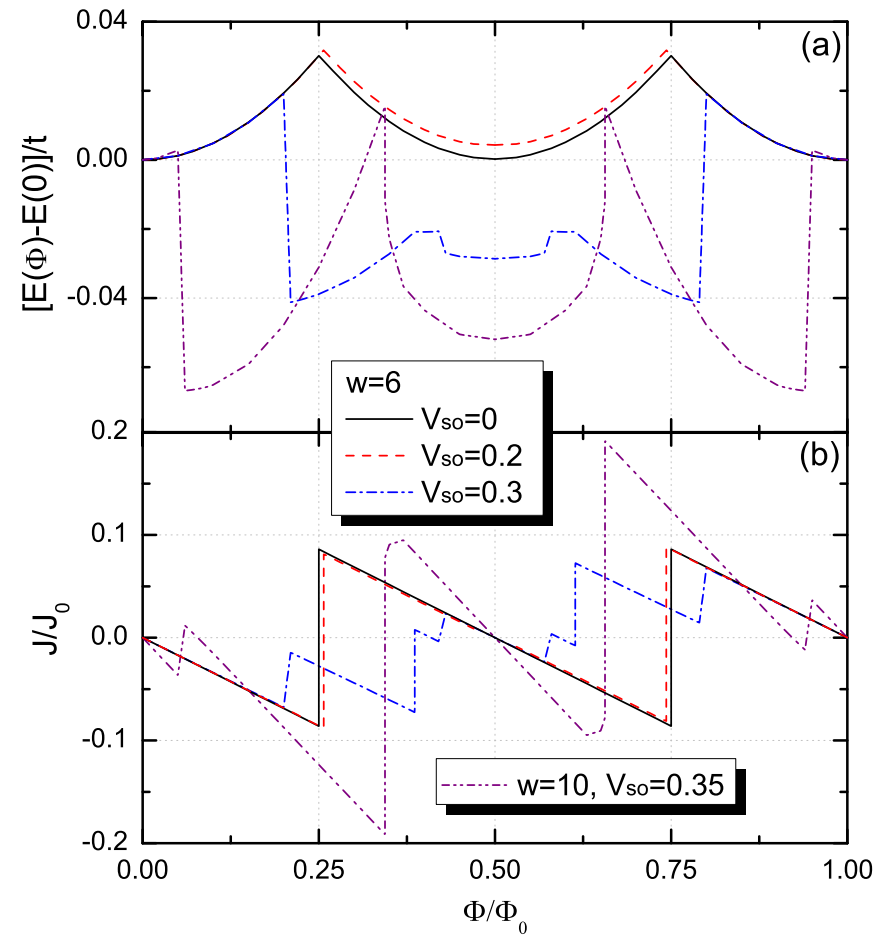

FIG. 2. (Color online) Total energy (a) and total supercurrent (b) for a square $40 \times 40$ loop with $\mathrm{SO}$ coupling strength $V_{\mathrm{so}}=0$ (black solid curves), $V_{\mathrm{so}}=0.2$ (red dashed curves), and $V_{\mathrm{so}}=0.3$ (blue dash-dotted curves) for an arm width $w=6$ and $V_{\mathrm{so}}=0.35$ (purple dash-dot-dotted curves) for $w=10$ as a function of magnetic flux $\Phi$. The pair interaction is $V=1.4$, and the temperature $T=0$.

$d$-wave pairing interaction is chosen as $V=1.4$. For a system with a large gap regime $V$ and without the SO interaction, the local minimum of the total energy at $\Phi=\Phi_{0} / 2$ approaches the energy value for $\Phi=0$, and the circulating supercurrent exhibits an almost $\Phi_{0} / 2$ periodicity, which is analogous to previous studies in Ref. [8]. With increasing $V_{\text {so }}$, the total energy still reaches a local minimum at $\Phi=\Phi_{0} / 2$, while the flux value of the $q$ jump shifts gradually from $\Phi=\Phi_{0} / 4$. Consequently, the current peaks become more asymmetric about the $J=0$ axis, and the deviation from a $\Phi_{0} / 2$-periodic behavior becomes larger.

To understand better the influence of the SO interaction on the magnetic flux periodicity, the eigenenergies near the Fermi energy $E_{F}=0$ (black dotted line) in the gap region corresponding to different $V_{\text {so }}$ as a function of flux $\Phi$ are shown in Fig. 3. Note that the zero-field order parameter $\Delta(\Phi=0) \approx$ 0.4 in the absence of SO coupling and slightly decreases with increasing $V_{\text {so }}$ for chosen pair interaction $V=1.4$. When $V_{\text {so }}=0$ [see Fig. 3(a)], a considerable empty spectral gap appears, and some well-separated discrete states are present due to the nodal character of the order parameter. Although the nearly $\Phi_{0} / 2$ flux periodicity, the regimes of condensate states with an even and an odd winding number $q$ of the order parameter, are still visible due to the mesoscopic effect. Notice that, seen in Fig. 3(b) for $V_{\text {so }}=0.2$, there is a distinct spin split phenomenon in the energy spectrum due to the breaking of the spin-reversal symmetry for particles and holes in the presence of the SO coupling effect. Besides the spin splitting,
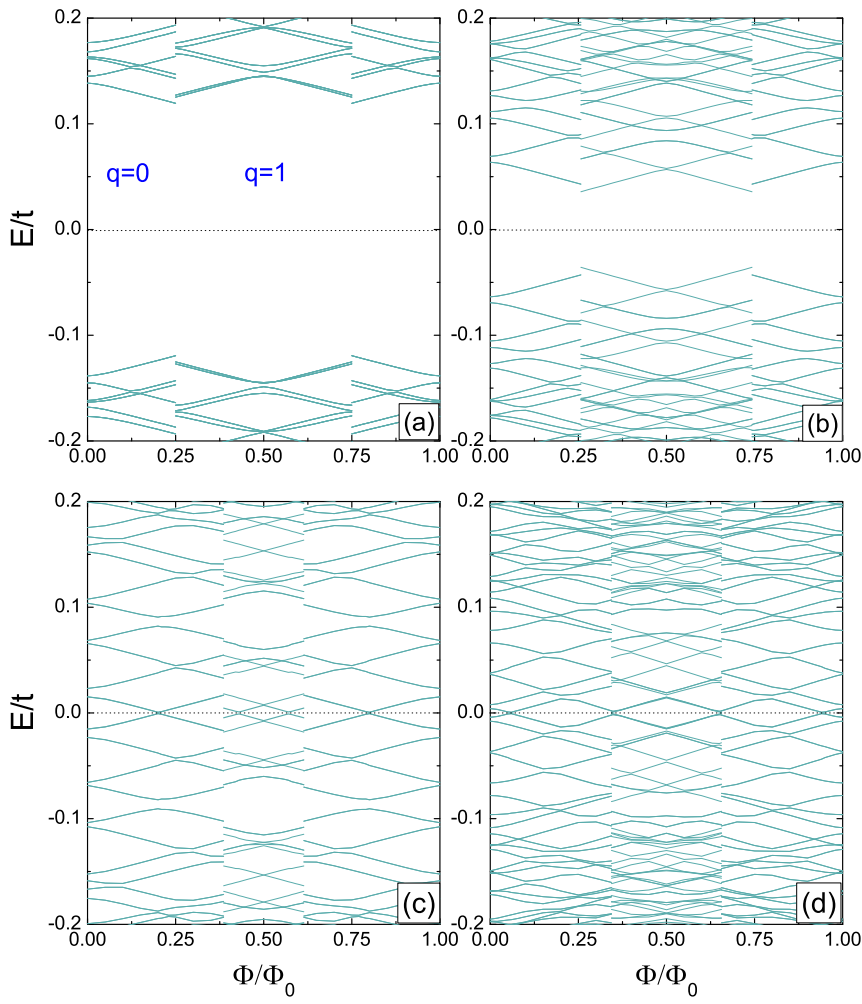

FIG. 3. (Color online) The eigenenergies in the gap region are shown for a square $40 \times 40$ loop with (a) $V_{\text {so }}=0$, (b) $V_{\text {so }}=0.2$, (c) $V_{\text {so }}=0.3$ for $w=6$, and (d) $V_{\text {so }}=0.35$ for $w=10$ as a function of flux $\Phi$. The black dotted line denotes the Fermi energy $E_{F}=0$. Reconstruction of the condensate takes place between even and odd $q$ regimes. The calculation is performed for $V=1.4$ and $T=0$.

there exists a destruction effect of the SO coupling on the superconducting pair potential. The split states are pushed toward the Fermi energy and the separation of spin-down and spin-up channels becomes remarkable as the SO coupling strength is increased. The change in the quasiparticle subgap states resulting from the SO coupling effect leads to stronger reconstruction of the energy spectrum of the condensate for different winding numbers of the order parameter. A small offset in the position of the $q$ transition takes place with respect to the flux value $(2 n+1) \Phi_{0} / 4$ (with integer $n$ ), which is the origin of the breaking of the $\Phi_{0} / 2$ periodicity in the ground-state energy and the supercurrent.

Obviously the energy evolution of superconducting states depends on the microscopic details of the energy spectrum. The states further away from $E_{F}$ provide most of the condensation energy, and the main contribution to the supercurrent arises from the occupied levels close to $E_{F}[1,8]$. For mesoscopic nodal superconducting loops with SO coupling, the density of states is finite close to the Fermi energy $E_{F}$, and allows for a flux dependent occupation probability. Likewise, the minima of the ground-state energy in the corresponding even and odd $q$ regime is also flux dependent. In the following we would like to analyze the effective ground state of $d$-wave loops for stronger SO interactions and focus on the flux interval $\Phi \in\left[0, \Phi_{0} / 2\right]$.

The blue dash-dotted curves in Fig. 2 show the total energy (a) and the total supercurrent (b) as a function of $\Phi$ for a 


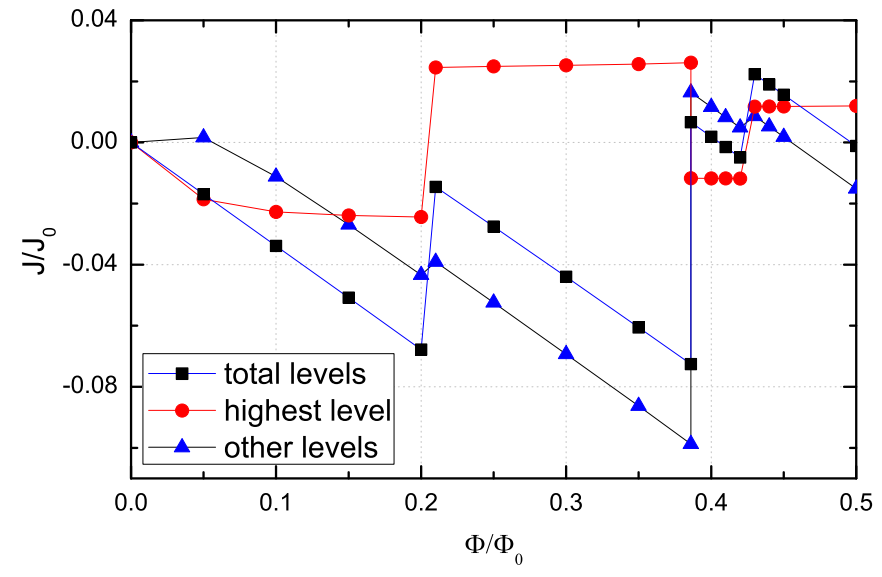

FIG. 4. (Color online) The contribution to the total supercurrent (black squares) arising from the level closest to $E_{F}$ (red circles) and other energy levels (blue triangles) for a square $40 \times 40$ loop with $w=6$ and $V_{\text {so }}=0.3$ in the flux regime $\left[0, \Phi_{0} / 2\right]$.

larger $V_{\mathrm{so}}=0.3$. We notice that the ground-state energy forms parabolas with minima at integer and half-integer values of $\Phi$, which is identical to the cases of weak $V_{\text {so }}$. Interestingly, between these flux values, there appear additional energy parabolas in $E(\Phi)$ and two abrupt energy drops at $\Phi=$ $0.21 \Phi_{0}$ and $0.43 \Phi_{0}$, where the superconducting ground state evolves between different parabolas. As a consequence, the supercurrents $J(\Phi)$ in Fig. 2(b) are discontinuous at the flux values where the energy jumps. Notice that the flux regimes belonging to different energy parabolas are not distributed equally. Nevertheless our results prove that new global minima in the total energy can emerge in the ground state, and the sawtooth pattern of supercurrent has a dominant Fourier component corresponding to fractional flux periodicity. Figure 3(c) presents the corresponding energy spectrum in the gap region for $V_{\mathrm{so}}=0.3$. We find that, compared to the case of weak SO coupling strength depicted in Fig. 3(b), the spin-split effect is enhanced when $V_{\text {so }}$ is further enlarged. In particular, the energy gap closes at several flux values which are exactly the same as the ones where the energy drops are found in Fig. 2(a). Notice that the energy level crossing the Fermi energy may originate from the nodal part of the spectrum. Figure 4 displays the contribution to the total supercurrent (black square curve) arising from different energy levels corresponding to the energy spectrum in Fig. 3(c). Clearly the level closest to $E_{F}$ contributes mainly to the current jumps in the total current. As shown by the curve with red circles in Fig. 4, the current changes sign at the crossing points with increasing flux, i.e., opposite orbital moments dominate around the crossing points. As a result, the sawtooth feature of flux-dependent supercurrent shows up. It is noted that the chosen grid size in our self-consistent calculation is optimal to obtain reliable results in minimal time. We checked the flux periodic evolution of the total energy and supercurrent for a wider square loop with $w=10$, which is depicted by purple dash-dot-dotted curves in Fig. 2. For a sufficiently strong SO interaction $V_{\text {so }}=0.35$, new produced minima are also found in the ground-state energy. Simultaneously, the energy gap is closed at the flux where the energy jumps in the spectrum, as shown in Fig. 3(d). Thus, an unusual flux periodicity is generated.

Notice that the emergence of energy level crossing the Fermi energy may support the existence of Majorana fermion states in mesoscopic loop systems in the presence of SO coupling. It has been demonstrated that strong SO interactions at the surface of the sample can lift the twofold spin degeneracy and induce mixed singlet-triplet state $[32,33]$. We checked the present system and found that the gap function has both a spin-singlet component and an additional spin-triplet one at the same time when the SO coupling is introduced. The spin-triplet pairing becomes stronger with increasing $V_{\text {so }}$. In this case, odd-frequency pair amplitudes may exist at the surface [43]. Moreover, Majorana fermion states and non-Abelian topological phase can occur in the edges of the superconducting system [43-47].

To examine the zero-energy Majorana edge mode, we calculate the local density of states (LDOS) for the loop sample with SO interactions. The LDOS is given by

$$
\rho_{\mathbf{i} \sigma}(E)=\sum_{n, \sigma} \operatorname{Im}\left[\left|u_{\mathbf{i} \sigma}^{n}\right|^{2} \delta\left(E_{n}-E\right)+\left|v_{\mathbf{i} \sigma}^{n}\right|^{2} \delta\left(E_{n}+E\right)\right],
$$

where the Dirac $\delta$ function $\delta(x)$ is taken as $\Gamma / \pi\left(x^{2}+\Gamma^{2}\right)$ with the quasiparticle damping $\Gamma=10^{-4}$. The LDOS is proportional to the local differential tunneling conductance which could be measured in a low-temperature scanning tunneling microscopic (STM) experiment. In Fig. 5 we depict the zero-energy LDOS (the upper panels) and the circulating current (the bottom panels) for the case with zero energy gap: a square $40 \times 40$ loop with $w=6$ and $V_{\text {so }}=0.3$ at $\Phi=0.21 \Phi_{0}$. The left, middle, and right panels show the case of spin-up, spin-down, and total spin, respectively. We can clearly see that the zero-energy modes for spin-up and spin-down electrons emerge in different edges of the loop in Figs. 5(a) and 5(b). The zero-bias peak conductance and the closing superconducting gap reflect the existence of Majorana fermions in our system [49]. Interestingly, unidirectional (a)

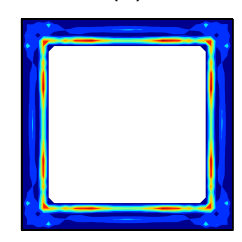

(d)

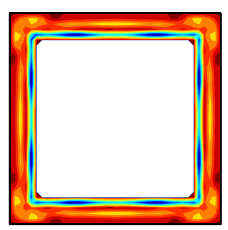

(b)

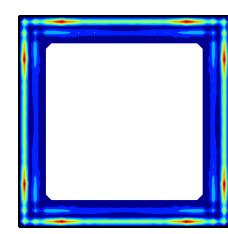

(e)

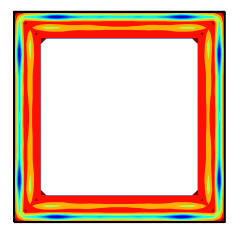

(c)

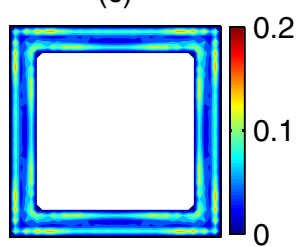

(f)

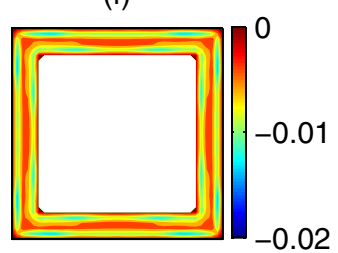

FIG. 5. (Color online) Zero-energy local density of states $\rho(E=$ $0)[(a)-(c)]$ and circulating current [(d)-(f)] for a square $40 \times 40$ loop with $V_{\mathrm{so}}=0.3$ and $w=6$ at the crossing point $\Phi=0.21 \Phi_{0}$. The left [(a) and (d)], middle [(b) and (e)], and right [(c) and (f)] panels correspond to the electrons with spin-up, spin-down, and total spin, respectively. 


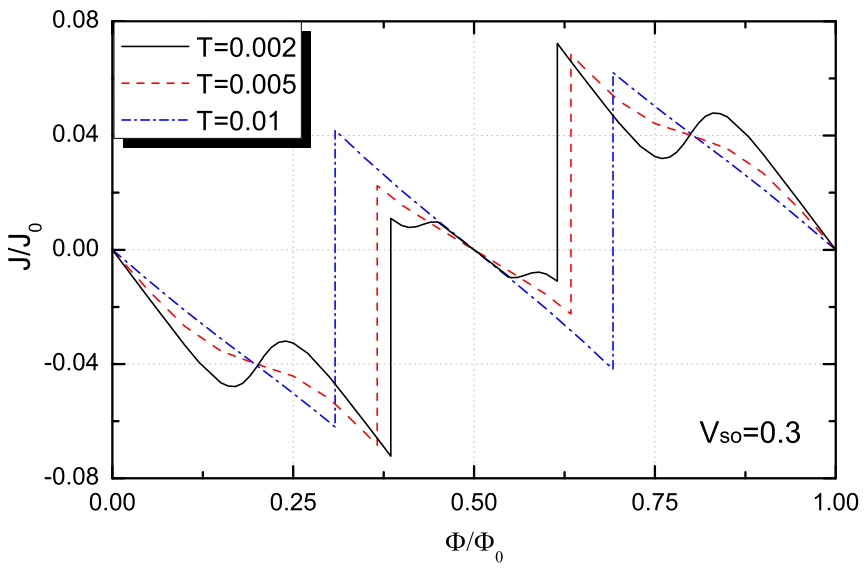

FIG. 6. (Color online) Total energy (a) and total supercurrent (b) for a square $40 \times 40$ loop with $w=6$ and $T=0.002$ (black solid curves), $T=0.005$ (red dashed curves), and $T=0.01$ (blue dashdotted curves) as a function of flux $\Phi$. The calculation is performed for $V=1.4$ and $V_{\text {so }}=0.3$.

moving edge modes which propagate in the same direction on opposite edges can appear in the loop system, as shown in Figs. 5(d) and 5(e). Notice that similar unidirectional Majorana edge states can be found in gapless topological superconductors with Rashba SO terms and an in-plane magnetic field in the presence of $p$ - or $s$-wave pairing [44].

Considering the finite temperature effect, we next investigate how the flux evolution depends on the temperature in the presence of the SO coupling effect. The flux dependence of the supercurrent for the loop with $w=6, V=1.4$, and $V_{\text {so }}=0.3$ at different temperatures $T$ below the critical temperature $T_{c}$ is displayed in Fig. 6. In contrast to the zero temperature case of Fig. 2 (blue dash-dotted curves), one can see that the oscillation patterns for the circulating current are very sensitive to the temperature. When the temperature increases, the additional peaks of the persistent supercurrent gradually disappears, i.e., the fractional current evolution is smoothed by the temperature effect. For an appropriate enlarged temperature, one can predict that the flux value of the $q$ jump is close to $\Phi=h c / 4 e$, which leads to an almost $\Phi_{0} / 2$ periodicity of the supercurrent [8]. Notice that, although the fractional periodic pattern of supercurrent is depressed with increasing $T$, the energy gap in the spectrum still vanishes at the same flux points as for the case of zero temperature in Fig. 3(c) (not shown here).

Finally, in order to investigate the effect of the geometry of our sample, we change the geometric symmetry of the system and consider the case of rectangular $d$-wave loops with parameter values: $w=6, V=1.4, V_{\text {so }}=0.3$, and $T=0$. The evolution of the total energy (a) and supercurrent (b) with magnetic flux for four rectangular loops is displayed in Fig. 7. The black solid curves are for the sample with $N_{x} \times N_{y}=38 \times 42$ and $N_{x x} \times N_{y y}=26 \times 30$, and the red dashed curves for the sample with $N_{x} \times N_{y}=36 \times 44$ and $N_{x x} \times N_{y y}=24 \times 32$. In comparison to the square system in Fig. 2, we still find an additional minimum in the ground-state energy near the flux value $\Phi_{0} / 4$ and the zigzag-like behavior of the flux-dependent current with increasing ratio of length to height $N_{y} / N_{x}$ of the rectangular loop (see the black solid

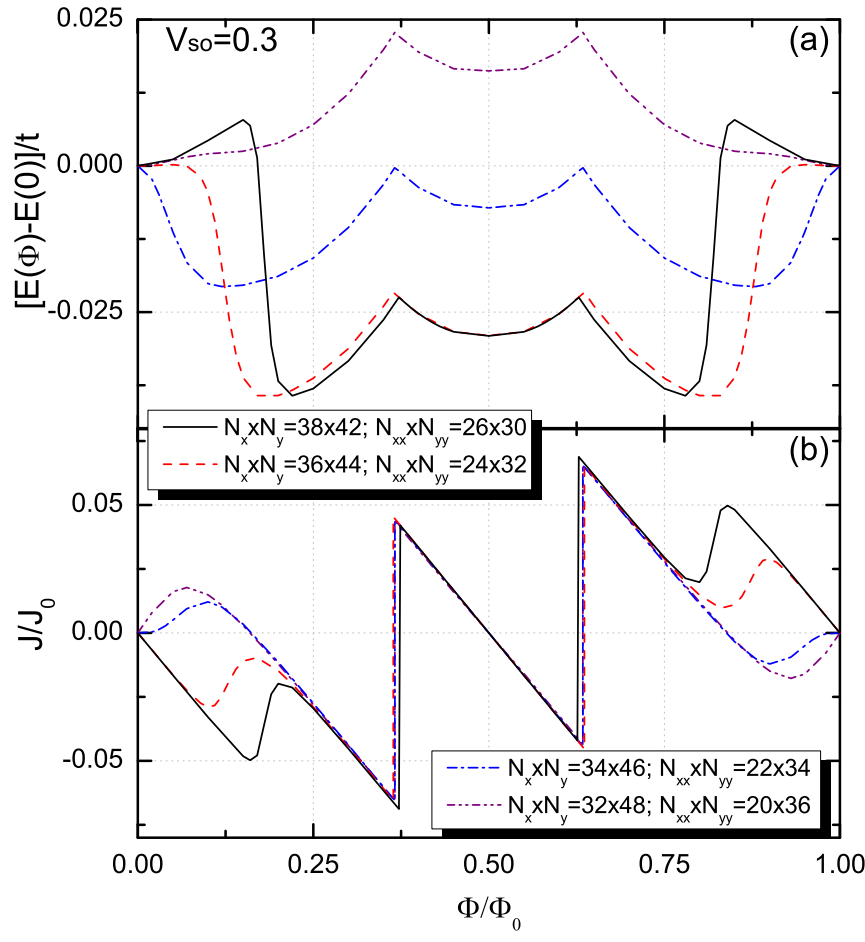

FIG. 7. (Color online) Total energy (a) and total supercurrent (b) for different rectangular loops with arm width $w=6$ as a function of flux $\Phi$. The calculation is performed for $V=1.4, V_{\text {so }}=0.3$, and $T=0$.

curves), while the jumps of energy and current behave more smoothly. For a critical ratio of $N_{y} / N_{x}$ (the red dashed curves), the jumps between different energy parabolas as shown in the square loop case nearly vanish. From the corresponding energy spectrum shown in Fig. 8(a), we find that an energy

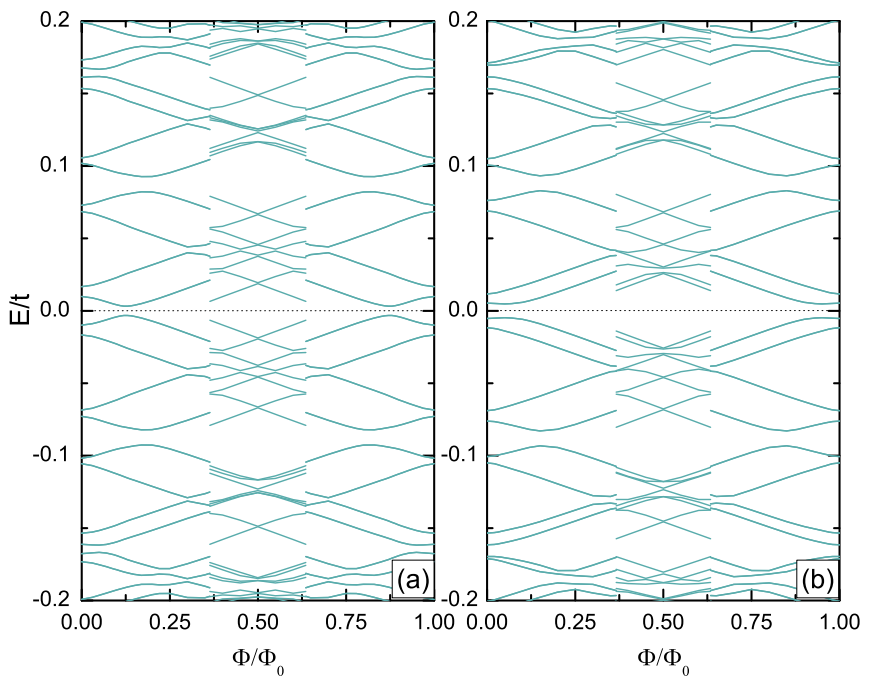

FIG. 8. (Color online) The eigenenergies in the gap region are shown for two rectangular samples with (a) $N_{x} \times N_{y}=36 \times 44$, $N_{x x} \times N_{y y}=24 \times 32$ and (b) $N_{x} \times N_{y}=32 \times 48, N_{x x} \times N_{y y}=$ $20 \times 36$ as a function of flux $\Phi$. The black dotted line denotes the Fermi energy $E_{F}=0$. The calculation is performed for $V=1.4$, $V_{\text {so }}=0.3$, and $T=0$. 
gap opens and the current-carrying state of the condensate are continuously changing near the crossing points. Consequently, the zero energy Majorana edge mode disappears. With further increasing the asymmetric ratio, the flux value where the minimum of the ground-state energy occurs in the $q=0$ regime will shift toward zero flux. In Fig. 7 we show also the results for two other rectangular loop cases with larger ratio $N_{y} / N_{x}$. The blue dash-dotted curves are for the sample with $N_{x} \times N_{y}=34 \times 46$ and $N_{x x} \times N_{y y}=22 \times 34$, and the purple dash-dot-dotted curves for the sample with $N_{x} \times N_{y}=$ $32 \times 48$ and $N_{x x} \times N_{y y}=20 \times 36$. We can see that the local minimum of the energy at $\Phi=0$ is evolved into a global one at some critical ratio. Meanwhile, enlarged $N_{y} / N_{x}$ can lead to opposite directions of the current around the zero flux value, which indicates that the occupied level closest to $E_{F}$ with an orbital moment opposite to the applied magnetic field starts to dominate. Figure 8(b) depicts the energy spectrum for the rectangular sample with $N_{x} \times N_{y}=32 \times 48$ and $N_{x x} \times N_{y y}=20 \times 36$. In contrast, we notice that the energy gap in the whole flux regime is enlarged. Simultaneously, the flux value where the highest occupied state approaches $E_{F}$ shifts toward $\Phi=0$. Consequently, a paramagnetic response is generated for small $\Phi$, as displayed in Fig. 7(b).

\section{CONCLUSIONS}

We have investigated the SO coupling effect on the flux dependence of the energy and supercurrent in mesoscopic $d$-wave superconducting loops by numerically solving the extended $\mathrm{BdG}$ equations self-consistently. The energy spectrum splits because the SO coupling affects the spin characteristics of the quasiparticles. Due to the reconstruction of the condensate and the flux-driven change of the quasiparticle low-energy states, we found a breaking of the $h c / 2 e$-periodic energy and supercurrent. For an appropriate SO coupling strength, zero-energy Majorana edge modes can be realized at the closing points of the energy gap. Simultaneously, energy jumps between different energy parabolas emerge in the ground state, which provides a possible mechanism to generate a fractional flux periodicity in the supercurrent. At finite temperature, the fractional periodic evolution with sawtooth pattern of the supercurrent can be smoothed with increasing $T$. In addition, for rectangular loops, the minimum of the total energy near the flux value $\Phi_{0} / 4$ disappears for a sufficiently large ratio of length to height, and a paramagnetic response with opposite direction of the screening current around zero flux can occur in such systems. We expect that our theoretical calculations will be useful to realize Majorana fermions and to interpret unconventional flux-dependent periodic oscillations in multiply connected mesoscopic superconducting systems. Also, our results provide an experimental setup to detect the Majorana zero mode and fractional flux periodicity in loop systems, since the strength of the SO coupling can be tuned by changing the applied electric field [50-52].

\section{ACKNOWLEDGMENTS}

This work was supported by National Natural Science Foundation of China under Grants No. 61371020 and No. 61271163, by Visiting Scholar Program of Shanghai Municipal Education Commission, by Innovation Program of Shanghai Municipal Education Commission under Grant No. 13YZ006, and by the Flemish Science Foundation (FWO-VI).
[1] F. Loder, A. P. Kampf, T. Kopp, J. Mannhart, C. W. Schneider, and Y. S. Barash, Nat. Phys. 4, 112 (2008).

[2] T.-C. Wei and P. M. Goldbart, Phys. Rev. B 77, 224512 (2008).

[3] V. Vakaryuk, Phys. Rev. Lett. 101, 167002 (2008).

[4] Y. S. Barash, Phys. Rev. Lett. 100, 177003 (2008).

[5] V. Juričić, I. F. Herbut, and Z. Tešanović, Phys. Rev. Lett. 100, 187006 (2008).

[6] F. Loder, A. P. Kampf, and T. Kopp, Phys. Rev. B 78, 174526 (2008).

[7] F. Loder, A. P. Kampf, T. Kopp, and J. Mannhart, New J. Phys. 11, 075005 (2009).

[8] G.-Q. Zha, M. V. Milosevic, S.-P. Zhou, and F. M. Peeters, Phys. Rev. B 80, 144501 (2009); 84, 132501 (2011).

[9] J.-X. Zhu and H. T. Quan, Phys. Rev. B 81, 054521 (2010).

[10] T. Lindstrom, S. A. Charlebois, A. Ya. Tzalenchuk, Z. Ivanov, M. H. S. Amin, and A. M. Zagoskin, Phys. Rev. Lett. 90, 117002 (2003).

[11] C. W. Schneider, G. Hammerl, G. Logvenov, T. Kopp, J. R. Kirtley, P. J. Hirschfeld, and J. Mannhart, Europhys. Lett. 68, 86 (2004).

[12] S. I. Koh, Phys. Rev. B 49, 8983 (1994).

[13] P. Tarasewicz, Eur. Phys. J. B 41, 185 (2004).
[14] A. A. Aligia, A. P. Kampf, and J. Mannhart, Phys. Rev. Lett. 94, 247004 (2005).

[15] E. Berg, E. Fradkin, and S. A. Kivelson, Phys. Rev. B 79, 064515 (2009).

[16] E. Berg, E. Fradkin, E.-A. Kim, S. A. Kivelson, V. Oganesyan, J. M. Tranquada, and S. C. Zhang, Phys. Rev. Lett. 99, 127003 (2007).

[17] D. F. Agterberg and H. Tsunetsugu, Nat. Phys. 4, 639 (2008).

[18] D. F. Agterberg, M. Sigrist, and H. Tsunetsugu, Phys. Rev. Lett. 102, 207004 (2009).

[19] E. Berg, E. Fradkin, and S. A. Kivelson, Nat. Phys. 5, 830 (2009).

[20] F. Loder, A. P. Kampf, and T. Kopp, Phys. Rev. Lett. 111, 047003 (2013).

[21] V. Vakaryuk and V. Vinokur, Phys. Rev. Lett. 107, 037003 (2011).

[22] J.-W. Huo, W.-Q. Chen, S. Raghu, and F.-C. Zhang, Phys. Rev. Lett. 108, 257002 (2012).

[23] J. Jang, D. G. Ferguson, V. Vakaryuk, R. Budakian, S. B. Chung, P. M. Goldbart, and Y. Maeno, Science 331, 186 (2011).

[24] L. F. Chibotaru, V. H. Dao, and A. Ceulemans, Europhys. Lett. 78, 47001 (2007); L. F. Chibotaru and V. H. Dao, Phys. Rev. B 81, 020502(R) (2010); P. J. Pereira, L. F. Chibotaru, and V. V. Moshchalkov, ibid. 84, 144504 (2011). 
[25] R. Geurts, M. V. Milosevic, and F. M. Peeters, Phys. Rev. B 81, 214514 (2010).

[26] J. C. Piña, C. C. de Souza Silva, and M. V. Milošević, Phys. Rev. B 86, 024512 (2012).

[27] G.-Q. Zha, Q. Wang, and S.-P. Zhou, J. Appl. Phys. 113, 213903 (2013).

[28] E. Babaev, Phys. Rev. Lett. 89, 067001 (2002).

[29] E. Babaev, Phys. Rev. Lett. 94, 137001 (2005).

[30] J. Garaud and E. Babaev, Phys. Rev. B 86, 060514 (2012).

[31] V. M. Edelstein, Phys. Rev. Lett. 75, 2004 (1995).

[32] L. P. Gorkov and E. I. Rashba, Phys. Rev. Lett. 87, 037004 (2001).

[33] V. V. Kabanov, Phys. Rev. B 69, 052503 (2004).

[34] B.-L. Gao and S.-J. Xiong, Phys. Rev. B 75, 104507 (2007).

[35] R. H. Silsbee, J. Phys.: Condens. Matter 16, R179 (2004).

[36] S. P. Zhou, Y. M. Shi, B. H. Zhu, and G. Q. Zha, Phys. Rev. B 73, 174503 (2006).

[37] H. Meng, H. W. Zhao, L. f. Zhang, L. M. Shi, G. Q. Zha, and S. P. Zhou, Europhys. Lett. 88, 17005 (2009).

[38] Y. Tanaka, Y. Mizuno, T. Yokoyama, K. Yada, and M. Sato, Phys. Rev. Lett. 105, 097002 (2010).

[39] K. Yada, M. Sato, Y. Tanaka, and T. Yokoyama, Phys. Rev. B 83, 064505 (2011).
[40] A. P. Schnyder and S. Ryu, Phys. Rev. B 84, 060504(R) (2011).

[41] P. M. R. Brydon, A. P. Schnyder, and C. Timm, Phys. Rev. B 84, 020501(R) (2011).

[42] A. P. Schnyder, P. M. R. Brydon, and C. Timm, Phys. Rev. B 85, 024522 (2012).

[43] Y. Tanaka, M. Sato, and N. Nagaosa, J. Phys. Soc. Jpn. 81, 011013 (2012).

[44] C. L. M. Wong, J. Liu, K. T. Law, and P. A. Lee, Phys. Rev. B 88, 060504(R) (2013).

[45] M. Sato, Y. Takahashi, and S. Fujimoto, Phys. Rev. Lett. 103, 020401 (2009); Phys. Rev. B 82, 134521 (2010).

[46] J. D. Sau, R. M. Lutchyn, S. Tewari, and S. Das Sarma, Phys. Rev. Lett. 104, 040502 (2010).

[47] M. Sato and S. Fujimoto, Phys. Rev. Lett. 105, 217001 (2010).

[48] P. G. de Gennes, Superconductivity of Metals and Alloys (Addison-Wesley, New York, 1994).

[49] S. R. Elliott and M. Franz, arXiv:1403.4976.

[50] D. Grundler, Phys. Rev. Lett. 84, 6074 (2000).

[51] J. Nitta, T. Akazaki, H. Takayanagi, and T. Enoki, Phys. Rev. Lett. 78, 1335 (1997); T. Koga, J.nbsp;Nitta, T. Akazaki, and H. Takayanagi, ibid. 89, 046801 (2002).

[52] V. P. Mineev and K. V. Samokhin, Phys. Rev. B 72, 212504 (2005). 